\title{
Annexin A4 induces platinum resistance in a chloride-and calcium-dependent manner
}

\author{
Akiko Morimoto ${ }^{1,2}$, Satoshi Serada2, Takayuki Enomoto ${ }^{3}$, Ayako Kim², Shinya \\ Matsuzaki ${ }^{1}$,Tsuyoshi Takahashi' ${ }^{2,4}$,Yutaka Ueda ${ }^{1}$, Kiyoshi Yoshino ${ }^{1}$, Masami \\ Fujita $^{1}$, Minoru Fujimoto ${ }^{2}$, Tadashi Kimura ${ }^{1}$ and Tetsuji Naka ${ }^{2}$ \\ ${ }^{1}$ Department of Obstetrics and Gynecology, Osaka University Graduate School of Medicine, Japan \\ ${ }^{2}$ Laboratory for Immune Signals, National Institute of Biomedical Innovation, Japan \\ 3 Department of Obstetrics and Gynecology, Niigata University Medical School, Japan \\ ${ }^{4}$ Department of Surgery, Osaka University Graduate School of Medicine, Japan \\ Correspondence to: Tetsuji Naka, email: tnaka@nibio.go.jp \\ Keywords: Annexin A4; platinum resistance; annexin repeat; chloride ion \\ Received: April 04, $2014 \quad$ Accepted: August 03, $2014 \quad$ Published: August 04, 2014
}

This is an open-access article distributed under the terms of the Creative Commons Attribution License, which permits unrestricted use, distribution, and reproduction in any medium, provided the original author and source are credited.

\section{ABSTRACT}

Platinum resistance has long been a major issue in the treatment of various cancers. We previously reported that enhanced annexin A4 (ANXA4) expression, a $\mathrm{Ca}^{2+}$-regulated phospholipid-binding protein, induces chemoresistance to platinumbased drugs. In this study, we investigated the role of annexin repeats, a conserved structure of all the annexin family, responsible for platinum-resistance as well as the effect of knockdown of ANXA4. ANXA4 knockdown increased sensitivity to platinum-based drugs both in vitro and in vivo. To identify the domain responsible for chemoresistance, ANXA4 deletion mutants were constructed by deleting annexin repeats one by one from the $\mathrm{C}$ terminus. Platinum resistance was induced both in vitro and in vivo in cells expressing either full-length ANXA4 or the deletion mutants, containing at least one intact annexin repeat. However, cells expressing the mutant without any calcium-binding sites in the annexin repeated sequence, which is essential for ANXA4 translocation from the cytosol to plasma membrane, failed to acquire platinum resistance. After cisplatin treatment, the intracellular chloride ion concentration, whose channel is partly regulated by ANXA4, significantly increased in the platinum-resistant cells. These findings indicate that the calcium-binding site in the annexin repeat induces chemoresistance to the platinum-based drug by elevating the intracellular chloride concentration.

\section{INTRODUCTION}

Since cisplatin was first introduced as an anticancer drug in the 1970s [1], various platinum-based drugs have been developed and widely used not only against gynecological but also against other cancers, including lung, colorectal, testicular, prostate and bladder cancer [26]. Although these platinum-based drugs have significantly contributed to improve survival rates, chemoresistance to these drugs has become a major problem in recent years [7-9]. It has now been elucidated that the mechanism of platinum resistance is mediated by reduced platinum accumulation, increased platinum detoxification, increased repair of platinum-DNA adducts and inhibited apoptosis [10-12]. Several proteins have been reported to be candidate factors such as copper transporters: CTR1[13], ATP7A and ATP7B[14-17]; multidrug resistance protein 2 (MRP2) [18-20]; glutathione S-transferase enzyme $\pi$ (GST $\pi)$ [21]; excision cross-complementing gene 1 (ERCC1) [22]; receptor-interacting protein 1 (RIP1) [23]; microRNAs [24-26]; and p53 [27]. In contrast, there are still several proteins related to platinum resistance without a full understanding of how these proteins help cells to confer platinum-based drugs.

We recently reported that annexin A4 (ANXA4) is overexpressed in ovarian clear cell carcinoma and 
induces chemoresistance to platinum-based drugs [28]. Annexins are calcium-regulated and negatively charged phospholipid membrane-binding proteins. The basic structure of annexins consists of 2 major domains: a conserved structural element called an annexin repeat, a segment of 70 amino acid residues at the $\mathrm{C}$ terminus, and the $\mathrm{N}$-terminal region unique for a given member of the family and determining individual annexin properties in vivo. The annexin repeat possesses the calcium and membrane binding sites and is responsible for mediating the canonical membrane binding properties [29]. These domains in ANXA4 are surrounded by relatively short amino and carboxy termini that do not have any known function [30]. ANXA4 is involved in membrane permeability, exocytosis and regulation of chloride channels in a calcium-dependent manner [29, 31-33]. ANXA4 is almost exclusively expressed in epithelial cells [34]. With regard to cancer, ANXA4 overexpression has been reported in various tumours, such as lung, gastric, colorectal, renal, pancreatic, ovarian and prostate cancer [28, 35-39] and is associated with tumour invasiveness, metastasis and chemoresistance [37, 40]. Moreover, ANXA4 has been shown to be associated with resistance to platinum-based drugs [28, 41-43].

ANXA4-induced platinum resistance appears to be mediated in part by the increased extracellular efflux of platinum mediated by the copper transporter ATP7A[28, 44]. Another mechanism of ANXA4induced chemoresistance is the modulation of $N F-\kappa B$ transcriptional activity [45]. ANXA4 suppresses NF- $\kappa \mathrm{B}$ transcriptional activity through interaction with the p50 subunit in a calcium-dependent manner; ANXA4 causes resistance to apoptosis induction by etoposide.

While ANXA4 prominently associated with chemoresistance, the functional domain of ANXA4 remains unclear. Therefore, to clarify the functional domain of ANXA4 is required to understand detailed mechanisms of the chemoresistance induced by ANXA4 and also overcome chemoresistance. In this study, focusing on platinum resistance, we aimed to identify the ANXA4 domain relevant to chemoresistance with regard to its structure as well as to test whether knockdown of ANXA4 expression could improve platinum resistance. Our data showed that the annexin repeat plays an important role in platinum resistance induced by ANXA4, which occurs in a calcium-dependent manner.

\section{RESULTS}

\section{Establishment of ANXA4 knockdown RMG-I cells}

To create cell lines with a stable ANXA4 knockdown, we analysed ANXA4 expression in ovarian cancer cells using western blotting. ANXA4 expression was strong in clear cell carcinoma cell lines (OVTOKO, OVISE and RMG-I) compared with serous adenocarcinoma cell lines (A2780, OVCAR3 and OVSAHO) and a mucinous adenocarcinoma cell line (MCAS; Fig. 1A). To see whether blocking ANXA4 expression was a valid chemosensitising strategy for ovarian clear cell carcinoma treatment, ANXA4 was stably suppressed using an ANXA4 shRNA plasmid. We established RMG-I-Y4 and R5 cell clones as well as RMG-I NC7 cell clones transfected with the empty vector as a control. Compared with RMG-I NC7 and untransfected control parent RMG-I cells, ANXA4 expression was markedly down-regulated at the protein level in RMG-I-Y4 and RMG-I-R5 cells (Fig. 1B). In the absence of any drug treatment, the growth rate among the 4 cell lines was similar in vitro (data not shown).

\section{Knockdown of ANXA4 expression enhances sensitivity to cisplatin and carboplatin}

The sensitivity to cisplatin and carboplatin was assessed in the 3 RMG-I clones NC7, R5 and Y4. Compared with the $\mathrm{IC}_{50}$ for cisplatin in NC7 cells, $\mathrm{IC}_{50}$ was significantly decreased in Y4 cells and $\mathrm{R} 5$ cells $(p<$ 0.01; Fig. 1C, left panel). Similarly, $\mathrm{IC}_{50}$ for carboplatin significantly decreased in $\mathrm{Y} 4$ cells and R5 cells compared with NC7 cells ( $p<0.01$; Fig. $1 \mathrm{C}$, right panel). $\mathrm{IC}_{50}$ for cisplatin and carboplatin decreased approximately 2 -fold because of the knockdown of ANXA4 expression.

A

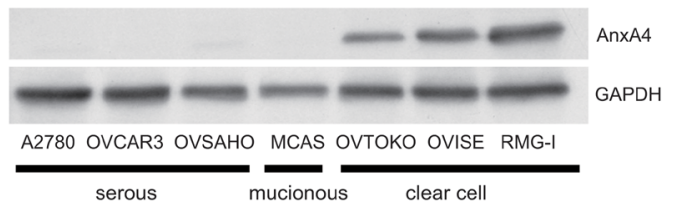

B

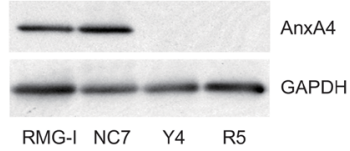

C
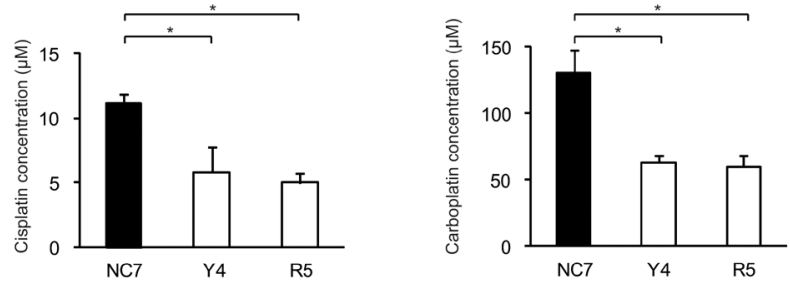

Fig.1: Knockdown of ANXA4 expression attenuates platinum resistance. (A) ANXA4 expression in indicated ovarian cancer cell lines and (B) established ANXA4 knockdown RMG-I cells (R5 and Y4) was confirmed using Western blotting. (C) $\mathrm{IC}_{50}$ for both cisplatin and carboplatin was significantly reduced in R5 and Y4 cells compared with NC7 cells. Data are presented as mean $\pm \mathrm{SD}(* p<0.01)$. 


\section{Suppression of ANXA4 expression improves platinum sensitivity in vivo}

To determine whether ANXA4 knockdown in clear cell carcinoma cells improved platinum sensitivity in vivo, $\mathrm{NC} 7$ and $\mathrm{Y} 4$ cells were subcutaneously injected in ICR $n u / n u$ mice. One week after inoculation with the tumour cells, the mice were randomised into 2 groups and received cisplatin or PBS i.p. twice a week for 4 weeks. The tumour growth rate in the absence of drugs was similar for both cell lines (Figs. 2A and 2B). Cisplatin treatment had very little effect on NC7 cells (Fig. 2A), but tumour volume markedly decreased in Y4 cells (Fig. 2B). Cisplatin treatment significantly decreased tumour growth in Y4 cells $(87.4 \pm 1.8 \%)$ compared with NC7 cells (-1.1 $\pm 18.0 \%$; $p<0.01$; Fig. 2C). These results showed that ANXA4 knockdown in the RMG-I cell line significantly attenuated resistance to cisplatin in vivo.

\section{The annexin repeat domain is required for the platinum drug resistance}

To identify a possible resistance-related domain within the annexin repeated sequence of ANXA4, we constructed 3 deletion mutants by deleting the annexin

A

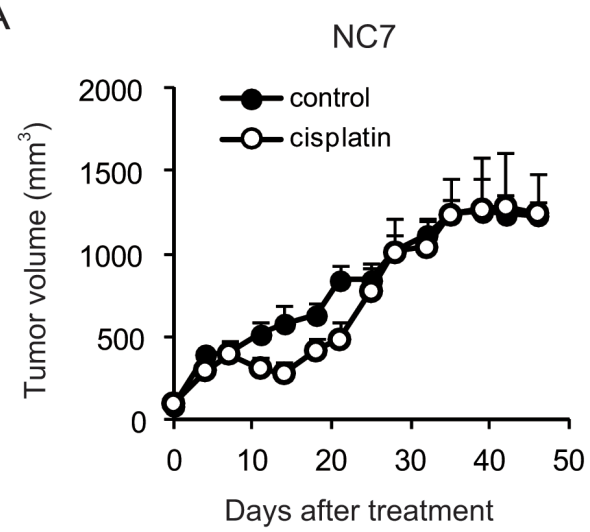

C

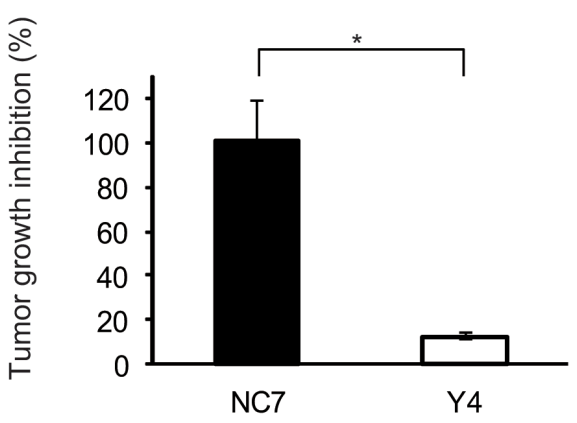

repeats one by one from the C-terminal region. Figure $3 \mathrm{~A}$ shows the structure of each deletion mutant. Full-length ANXA4, 3 ANXA4 deletion mutants or the empty vector were transfected into NUGC3 cells, whose endogenous ANXA4 expression is relatively low (Supplementary Fig. S1). Therefore, we established cell lines stably overexpressing full-length ANXA4 (FL-22), each ANXA4 deletion mutant (R3-6, R2-13 or R1-12) or the empty vector (NC-14). Expression of each ANXA4 deletion mutant was confirmed using Western blotting (Fig. 3B).

Subsequently, the sensitivity to the platinumbased drugs cisplatin and carboplatin was assessed. Cells transfected with full-length ANXA4 and the 3 deletion ANXA4 mutants were significantly more resistant to both cisplatin and carboplatin compared with control cells, approximately with a 1.7 - to 2.2 -fold increase in $\mathrm{IC}_{50}$ for cisplatin $(p<0.01)$ and a 1.4- to 1.7 -fold increase in $\mathrm{IC}_{50}$ for carboplatin $(p<0.05$; Fig. $3 \mathrm{C})$.

To test whether these deletion mutants induce platinum resistance through regulating cellular drug concentration as previously reported [28], we quantitated the intracellular platinum content of each deletion mutant-transfected cell clone after cisplatin treatment, which is one of the most representative platinum drugs. Platinum accumulation was significantly reduced in cells overexpressing either full-length ANXA4 or any of the 3

B

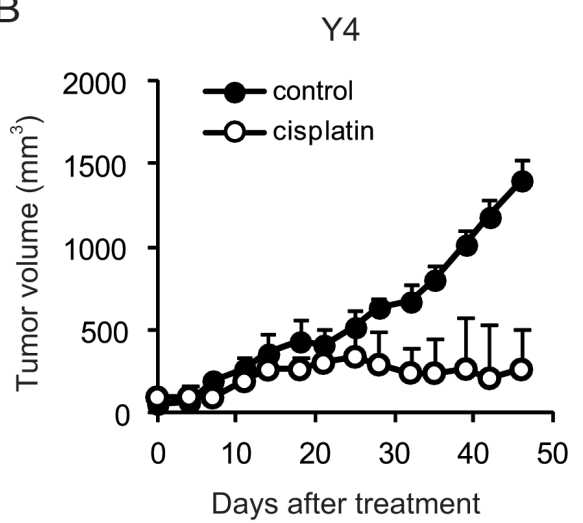

Fig.2: ANXA4 knockdown cells show enhanced sensitivity to cisplatin in vivo. Female ICR nu/nu mice were subcutaneously inoculated with RMG-I NC7 or Y4 cells and given PBS (control group: filled circles) or cisplatin i.p. (3 mg/kg; treatment group: open circles) twice weekly for 4 weeks ( $\mathrm{n}=6$ per group). Growth curves of NC7 tumours (A) and Y4 tumours (B). The mean volume (points) \pm SE (bars) is shown. (C) Comparison of the cisplatin-induced growth inhibition of tumours 46 days after treatment among NC7 and Y4 tumours. The average (columns) $\pm \mathrm{SE}$ (bars) are shown $(* p<0.01)$. 
deletion mutants compared with NC-14 cells regardless of the incubation time after cisplatin exposure (Fig. 3D). These results suggested that the decreased intracellular platinum contents were associated with platinum resistance of the cells transfected with ANXA4 full length and each deletion mutant.

\section{The calcium-binding site of the annexin repeat is responsible for platinum resistance}

As specified above, platinum resistance was enhanced in cells overexpressing ANXA4 deletion mutants, which contained at least 1 intact annexin repeat. Thus, to assess whether the calcium-binding site of the

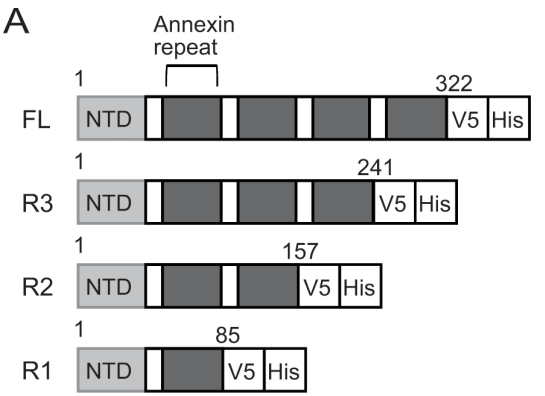

C

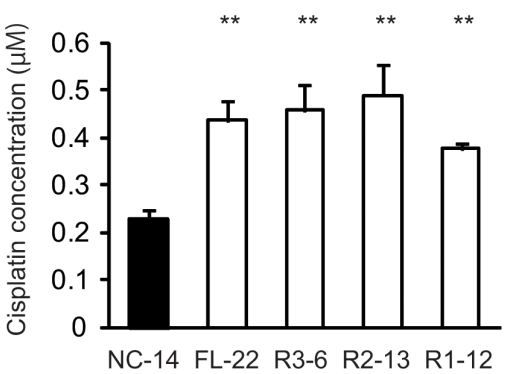

D

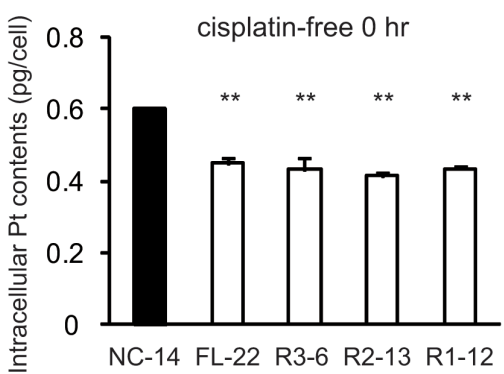

annexin repeat sequence was involved in chemoresistance, another deletion mutant, R1(E70A) was constructed. Within the annexin repeat next to the N-terminal region, the 70th amino acid, glutamic acid, was responsible for the calcium-dependent activity of ANXA4 [30]. Accordingly, at this site, the point mutation variant of R1, R1(E70A), loses the function of its calcium-binding site (Fig. 4A). Similar to other deletion mutants, R1(E70A) was transfected into NUGC3 cells and designated R1(E70A)-95. Western blotting revealed that R1-12 had the same molecular weight as R1(E70A)-95 (Fig. 4B). R1(E70A)-95 did not induce resistance to either cisplatin or carboplatin (Fig. 4C). Moreover, the intracellular platinum content of R1(E70A)-95-transfected cells did not decrease compared with that of NC-14 cells after $0 \mathrm{hr}$

B
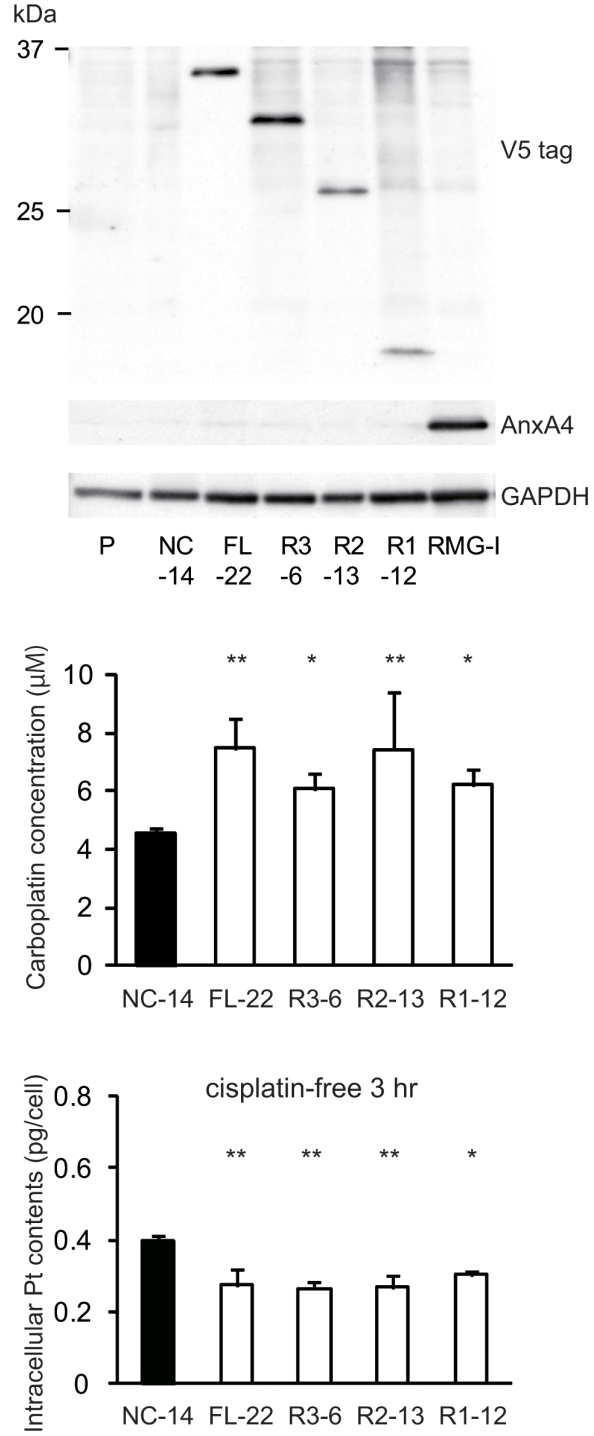

Fig.3: Annexin repeat domain is required for the platinum drug resistance. (A) A structural map of ANXA4 and 3 deletion mutant proteins. Annexin repeats were deleted one by one from the C-terminal site. (B) Established deletion mutant cells together with parent cells, control cells and RMG-I as a positive control were confirmed using Western blotting. (C) Compared with NC-14 cells, IC ${ }_{50}$ for both cisplatin and carboplatin was significantly increased in FL-22 and all other mutant cells. (D) Intracellular platinum accumulation after treatment with $100 \mu \mathrm{M}$ cisplatin for $60 \mathrm{~min}$ with or without additional $3 \mathrm{hr}$ of incubation in cisplatin-free medium. Data are presented as mean $\pm \mathrm{SD}(* p<0.05, * * p<0.01)$. 
or $3 \mathrm{hr}$ of additional incubation in cisplatin-free medium (Fig. 4D). According to the above results, the platinum resistance of ANXA4 seemed to be related to the calciumbinding site of the annexin repeat next to the N-terminal domain.

\section{The calcium-binding site of the annexin repeated sequence is required for the resistance to platinum-based drugs in vivo}

To determine whether the annexin deletion mutants of ANXA4 influenced the sensitivity to cisplatin in vivo,

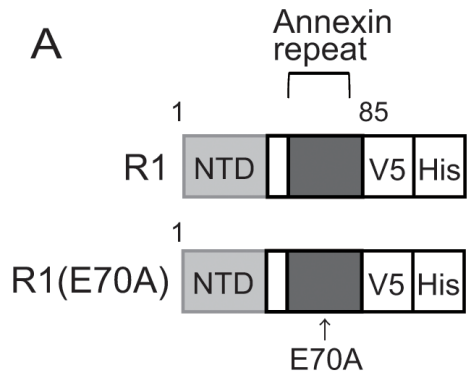

C

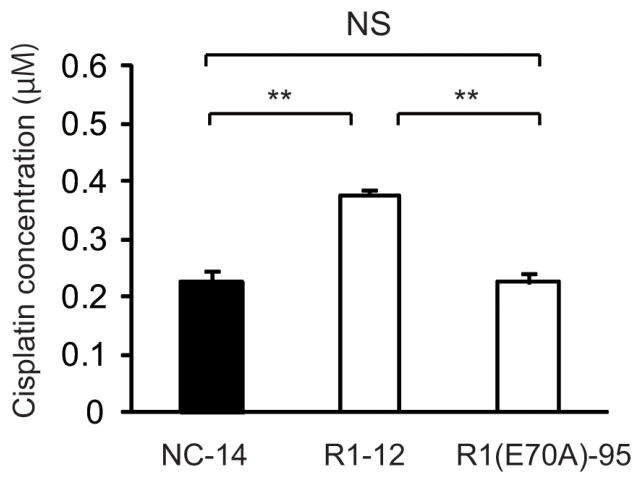

$\mathrm{D}$

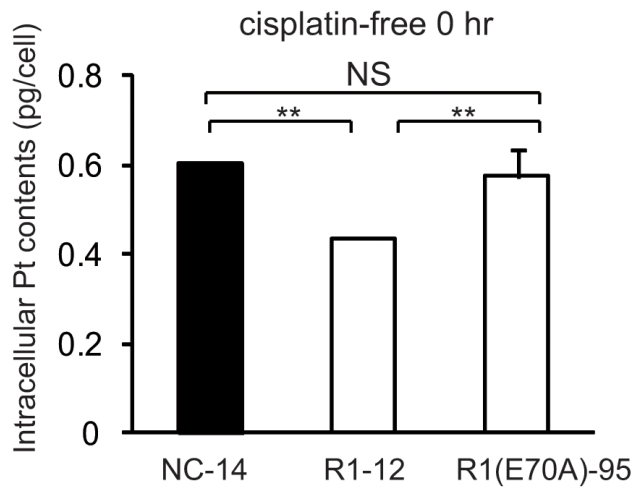

we inoculated ICR $n u / n u$ mice with NC-14, FL-22, R1-12 or R1(E70A)-95 cells. Mice in each group were randomised into 2 subgroups and received either cisplatin at $3 \mathrm{mg} /(\mathrm{kg} \cdot \mathrm{d})$ or PBS i.p. once a week for 3 weeks. Cisplatin markedly decreased tumour volume in mice injected with NC-14 and R1(E70A)-95 cells, whereas the treatment effect was relatively smaller in mice injected with FL-22 and R1-12 cells (Fig. 5A). Consistent with the tumour volume, tumour growth was significantly inhibited by cisplatin in mice inoculated with NC-14 (96.5 $\pm 1.3 \%)$ and R1(E70A)-95 cells $(87.8 \pm 11.4 \%)$ compared with those injected with FL-22 (48.5 $\pm 11.7 \%)$ and R1-12 cells $(37.7 \pm 9.8 \% ; p<0.01$; Fig. 5B). These in vivo results

B

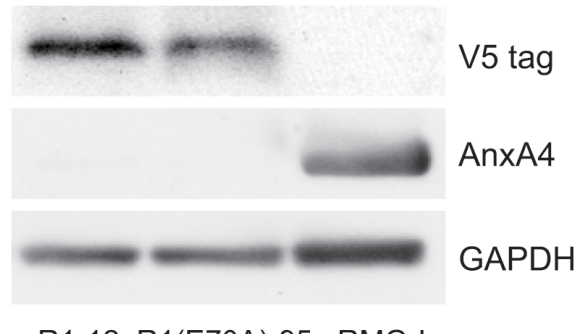

R1-12 R1(E70A)-95 RMG-I
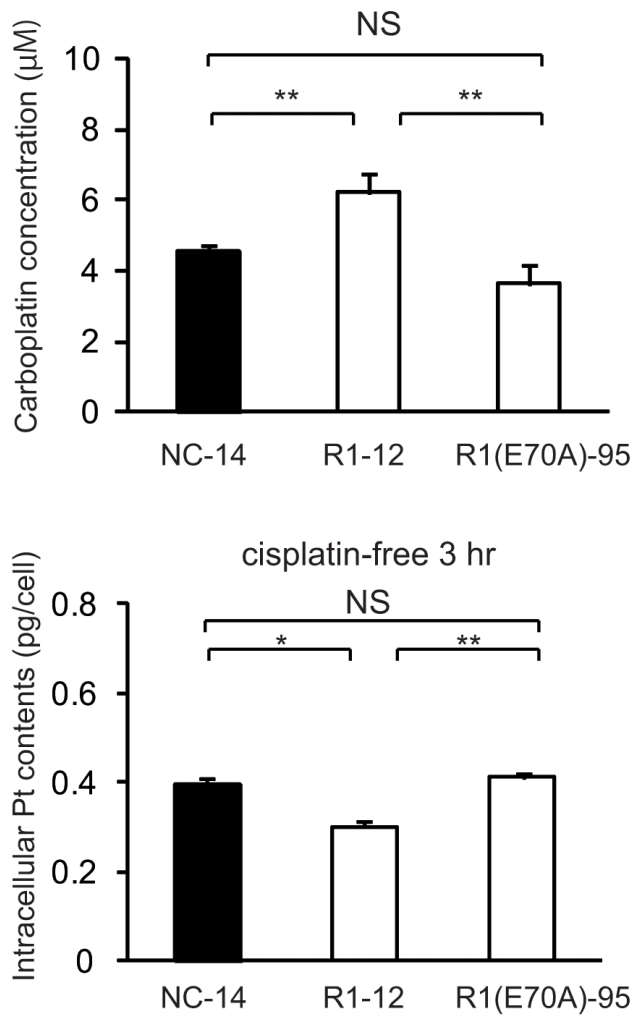

Fig.4: The calcium-binding site of the annexin repeat is responsible for induction of the platinum resistance. (A) A structural map of R1 and R1(E70A) variants without a function for the calcium-binding site. (B) Western blotting confirmed the mutant established cell lines. (C) Unlike R1-12, the R1(E70A)-95 cell clone was not resistant to either cisplatin or carboplatin. (D) Intracellular platinum accumulation in $\mathrm{R} 1(\mathrm{E} 70 \mathrm{~A})$ cells was the same as in $\mathrm{NC} 14$ cells both with or without additional $3 \mathrm{hr}$ of incubation in cisplatin-free medium. Data are presented as mean $\pm \mathrm{SD}(* p<0.05, * * p<0.01)$. 
were consistent with those obtained in vitro.

\section{Increase of the intracellular chloride concentration is related to cisplatin resistance}

To elucidate the molecular mechanisms of chemoresistance induced by ANXA4, we focused on the chloride channel because one of the functions of ANXA4 is inhibition of calcium-dependent chloride conductance[32]. According to the literature, treatment with cisplatin induces an increase of the intracellular $\mathrm{Ca}^{2+}$ concentration [46], which is an important ion for the phospholipid membrane-binding activity of ANXA4. In contrast, cisplatin exposure also induces an elevation of the intracellular chloride concentration: $\left[\mathrm{Cl}^{-}\right]_{\mathrm{i}}[47]$. Elevation of $\left[\mathrm{Cl}^{-}\right]_{\mathrm{i}}$ has been shown to prevent the aquation

A

NC-14

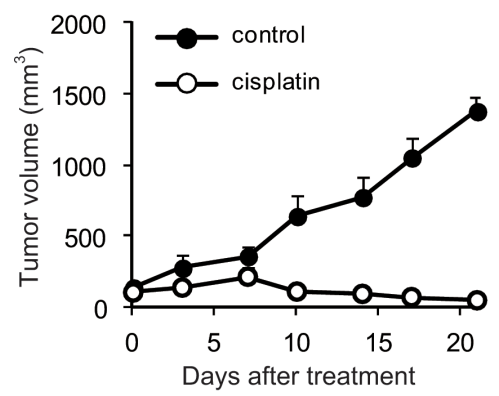

$\mathrm{R} 1-12$

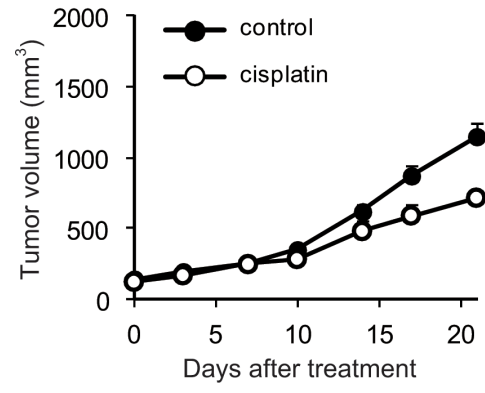

B

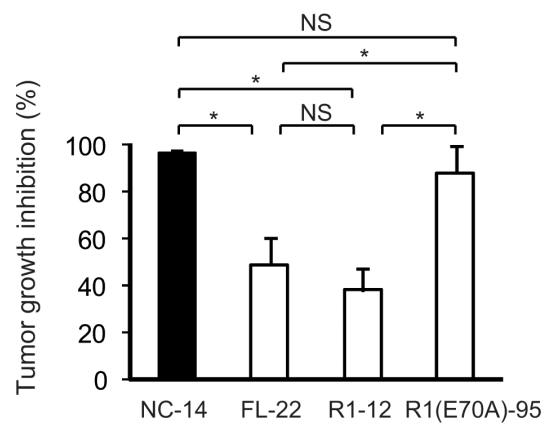

of 1 or 2 of the 2 chloride coordination sites in cisplatin, and only the aquated forms of cisplatin covalently bind to DNA. Nevertheless, the mechanisms of $\left[\mathrm{Cl}^{-}\right]$ i elevation because of cisplatin treatment have not been fully elucidated. We hypothesised that an increase in intracellular $\mathrm{Ca}^{2+}$ concentration after cisplatin exposure would result in translocation of ANXA4 from the cytosol to plasma membrane, which leads to $\left[\mathrm{Cl}^{-}\right]_{\mathrm{i}}$ accumulation through inhibition of the chloride channel by the $\mathrm{Ca}^{2+}-$ bound ANXA4. To confirm this hypothesis, we quantified $\left[\mathrm{Cl}^{-}\right]_{\mathrm{i}}$ after cisplatin treatment using MAQE fluorescence, a fluorescent $\mathrm{Cl}^{-}$indicator. Relative fluorescence was substituted for $\left[\mathrm{Cl}^{-}\right]_{\mathrm{i}}$ as previously reported [48].

We monitored MQAE fluorescence in control cells (NC-14), in cells overexpressing full-length ANXA4 (FL22) and in 2 deletion mutants (R1-12 and R1[E70A]-95). The relative fluorescence ratio before (F0) and after

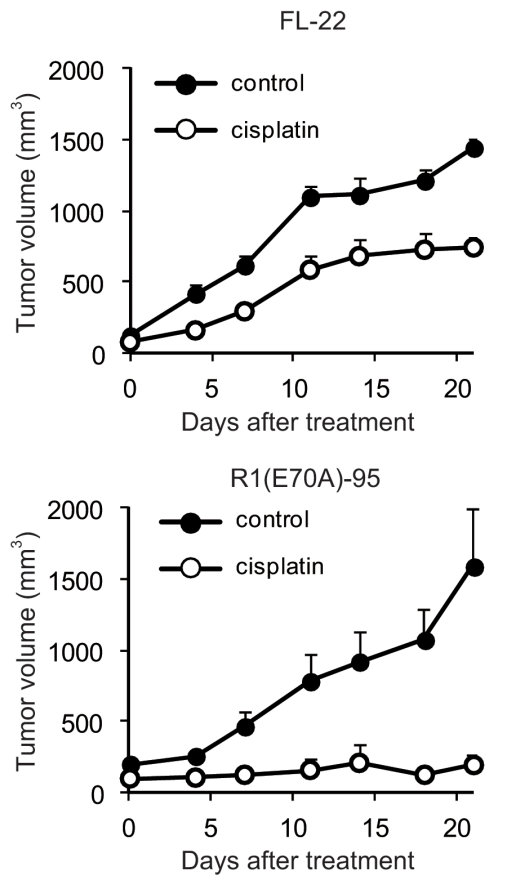

Fig.5: The calcium-binding site of the annexin repeat is required for platinum drug resistance in vivo. Female ICR nu/ $n u$ mice were subcutaneously inoculated with NC14, FL-22, R1-12 or R1(E70A)-95 cells and given PBS (control group: filled circles) or cisplatin i.p. ( $3 \mathrm{mg} / \mathrm{kg}$; treatment group: open circles) once a week for 3 weeks ( $=6$ per group). (A) Growth curves of tumours of each cell. The mean volume (points) $\pm \mathrm{SE}$ (bars) is shown. (B) Comparison of the cisplatin-induced growth inhibition of tumours 28 days after treatment. The average (columns) $\pm \mathrm{SE}$ (bars) are shown; ${ }^{*} p<0.01$. 
treatment with cisplatin for $30 \mathrm{~min}$ (F30) is shown in Figure 6. The inverse ratio of MQAE fluorescence 1/ (F30/F0), which is directly proportional to the increase in $\left[\mathrm{Cl}^{-}\right]_{\text {i }}$, was significantly elevated in the platinum-resistant cell clones FL-22 (1.12 \pm 0.03$)$ and R1-12 (1.12 \pm 0.01$)$ compared with sensitive clones, NC-14 (1.06 \pm 0.01$)$ and $\mathrm{R} 1(\mathrm{E} 70 \mathrm{~A})-95(1.06 \pm 0.02 ; p<0.01)$. Thus, the increase in $\left[\mathrm{Cl}^{-}\right]_{\mathrm{i}}$ is likely to be involved in cisplatin resistance.

\section{DISCUSSION}

ANXA4 has been reported to be strongly expressed and involved in chemoresistance in various cancers. The factors associated with ANXA4-induced chemoresistance have been reported, such as NF- $\kappa$ B [45] and ATP7A [44], but the structure of the protein, i.e. annexin repeats and calcium-binding sites in the annexin repeated sequence, has not been taken into account in relation to the ANXA4-induced chemoresistance. In this study, we showed that ANXA4 knockdown improved sensitivity to platinum drugs, and annexin repeats were involved in chemoresistance.

We first confirmed ANXA4 expression in various ovarian adenocarcinoma cell lines. As previously reported [28, 49], ANXA4 is significantly upregulated in clear cell carcinoma cell lines (OVTOKO, OVISE and RMG-I) compared with other histological types (serous and mucinous adenocarcinoma cell lines: A2780, OVCAR3, OVSAHO and MCAS). It has been previously demonstrated that enhanced ANXA4 expression induces platinum resistance both in vitro and in vivo [28, 44], but whether ANXA4 knockdown attenuates platinum resistance has been unknown thus far. Mogami et al. recently reported that an ANXA4 knockdown improves sensitivity to carboplatin in vitro using 2 cell lines of ovarian clear cell carcinoma, OVTOKO and OVISE. To the best of our knowledge, ours is the first study to show that ANXA4 knockdown markedly improves the sensitivity to platinum-based drugs not only in vitro but also in vivo (Figs. 1 and 2).

The result that ANXA4 knockdown improves sensitivity to platinum-based drugs suggests that ANXA4 is a good therapeutic target. To identify the functional domain(s) of ANXA4 that could be a promising therapeutic target, we focused on annexin repeats and constructed 4 deletion mutants (R3, R2, R1 and R1[E70A]). Resistance to platinum drugs was enhanced in cells transfected with mutants possessing at least 1 intact annexin repeat. In contrast, the sensitivity to platinum-based drugs improved among the R1(E70A)-transfected clones because in those cells, the calcium-binding site did not function properly (Figs. 3C and 4C). This result implies that the ANXA4induced chemoresistance to platinum-based agents is calcium dependent. It has been reported that cisplatin induced increase of intracellular calcium concentration in chemosensitive cells, but not in resistant cells $[46,50]$.
Together with this and the data by Chan et al., elevation of intracellular calcium concentration induced by cisplatin treatment may translocate $\mathrm{Ca}^{2+}$ bound form of ANXA4 from cytosol to plasma membrane, which results in platinum-resistance[32]. We are currently investigating on further analysis.

By analysing the intracellular platinum accumulation, we attempted to elucidate the mechanism of the platinum resistance induced by ANXA4 and its deletion mutants. When intracellular platinum contents were quantitated just after exposure to cisplatin or $3 \mathrm{hr}$ incubation with cisplatin-free medium after exposure to cisplatin, significantly less platinum accumulated in cells transfected with the full-length ANXA4 (FL-22) and 3 deletion mutants (R3-6, R2-13 and R1-12), all of which enhanced the resistance to the platinum-based drugs. In contrast, R1(E70A)-transfected cells (R1[E70A]-95), which did not induce chemoresistance, had the same amount of platinum accumulation as the control cells (Figs. 3D and 4D). These results suggest that the resistance to the platinum-based drugs is mediated by the decrease in intracellular platinum accumulation, which is calcium dependent. The annexin repeats, especially their calcium-binding sites, may be involved in inhibition of the influx, promotion of the efflux or both of platinum drugs. Recently, $\mathrm{Cu}$ transporters (CTR1 for the uptake and ATP7A and ATP7B for the efflux) have been reported to be involved in resistance to both cisplatin and carboplatin $[14,44,51]$. In addition, ANXA4 likely enhance platinum efflux through the interaction with ATP7A [44]. The possible mechanisms of inhibition of the influx mediated by ANXA4 remains unclear and further analyses are needed.

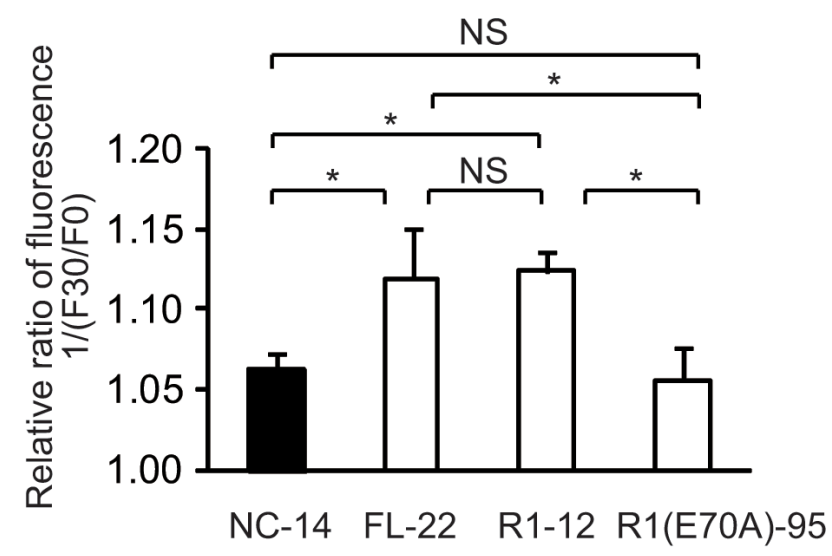

Fig.6: The increase of intracellular chloride concentration is related to cisplatin resistance. ANXA4 deletion mutant series cells (NC-14, FL-22, R1-12 and R1[E70A]-95) loaded with N-ethroxycarbonymethyl-6methoxyquinolinium bromide (MQAE) were exposed to $100 \mu \mathrm{M}$ cisplatin. The fluorescence pre-treatment and during treatment (30 min exposure) was compared in each cell clone. Data are presented as mean $\pm \mathrm{SD}(* p<0.01)$. 
Subsequently, the question regarding the involvement of calcium-binding site in the platinum resistance arose. To answer this question, we measured $\left[\mathrm{Cl}^{-}\right]_{\mathrm{i}}$ after cisplatin exposure. The significant increase in $\left[\mathrm{Cl}^{-}\right]_{\mathrm{i}}$ was observed in the cells with platinum resistance, FL-22 and R1-12, compared with cell clones without platinum resistance, NC-14 and R1(E70A)-95. In a previous study, higher $\left[\mathrm{Cl}^{-}\right]_{\mathrm{i}}$ was observed in cisplatinresistant cells compared with sensitive cells, whereas the intracellular cisplatin accumulation showed the opposite pattern [47]. These results, in addition to the results of decreased platinum accumulation in resistant mutants, indicate that ANXA4 induces platinum resistance through cellular drug efflux partly by elevating the intracellular chloride concentration. We report 'partly' because only cisplatin, not carboplatin, was tested in our $\left[\mathrm{Cl}^{-}\right]_{\mathrm{i}}$ assay. Cisplatin becomes intracellularly activated by the aquation of 1 or 2 of the 2 chloride coordination sites, but carboplatin does not contain any chloride coordination sites [1, 52-54]. Thus, this mechanism of resistance through elevation of $\left[\mathrm{Cl}^{-}\right]_{\mathrm{i}}$ may be specific to cisplatin and may not be true of carboplatin resistance. In this study, 3 cell clones overexpressing a deletion mutant (R3-6, R213, and R1-12) show stronger tolerance to cisplatin than to carboplatin in terms of their $\mathrm{IC}_{50}$; a 1.7- to 2.2-fold increase for cisplatin and only a 1.4- to 1.7 -fold increase for carboplatin (Fig. 3C). It is assumed that the increase in $\left[\mathrm{Cl}^{-}\right]_{\mathrm{i}}$ is one of the factors inducing cisplatin resistance.

In this study, the calcium-binding site in the annexin repeat next to the $\mathrm{N}$ terminus was observed to be responsible for the resistance to the platinum drugs. Nevertheless, the role of the other 3 calcium-binding sites has not yet been investigated. The roles of individual calcium-binding sites were demonstrated using sitedirected mutagenesis by Nelson and Creutz regarding calcium-dependent membrane binding and aggregation [30]. The mutations in each domain had different effects on the binding or aggregating activities, i.e. a mutation in the first or fourth domain had a greater effect on membrane binding. A mutation in the second domain had a stronger effect on membrane aggregation, whereas the mutation of the third domain was almost silent. Although the mechanisms involved in membrane binding/ aggregation and the mechanisms of chemoresistance are likely different, our data could provide some clues to understanding the function of each annexin repeat and each calcium-binding site in chemoresistance.

ANXA4 has been shown to induce resistance to paclitaxel and platinum-based drugs [55]. The effect of ANXA4 knockdown on paclitaxel sensitivity was assessed in a previous study. The effect of sensitivity to paclitaxel varied among different cell clones: ANXA4 knockdown in the OVTOKO cell line with acidic isoelectric point (IEPs) did not improve the sensitivity to paclitaxel, whereas OVISE cell lines with basic IEPs showed improved sensitivity to paclitaxel [43]. In our own preliminary data, significant chemosensitisation to paclitaxel and etoposide was confirmed in RMG-I Y4 and R5 (data not shown). Further studies are required to identify the detailed mechanism.

In summary, in this study, we observed the annexin repeat, especially its calcium binding site, was associated with platinum-resistance induced by ANXA4, and it happened in calcium-dependent manner. Our findings may help to understand the mechanisms of platinum resistance induced by other annexin family proteins, which possesses the same annexin repeat structure, and offer new strategies for the treatment of chemoresistant cancers.

\section{METHODS}

\section{Cell lines and culture}

The human ovarian serous adenocarcinoma cell line (OVSAHO), human ovarian mucinous adenocarcinoma cell line (MCAS), human ovarian clear cell carcinoma cell lines (OVTOKO, OVISE and RMG-I) and human gastric cancer cell line (NUGC3) were obtained from the Japanese Collection of Research Bioresources (Osaka, Japan). A2780 cells from the human ovarian serous adenocarcinoma were obtained from the European Collection of Animal Cell Culture (Salisbury, Scotland) and OVCAR-3 cells from another human ovarian serous adenocarcinoma were from American Type Culture Collection (Manassas, VA). MCAS cells were maintained in the DMEM medium and the others in the RPMI medium, all supplemented with $10 \%$ foetal bovine serum (FBS; Serum Source International, NC, USA) and 1\% penicillin-streptomycin (Nacalai Tesque, Kyoto, Japan) at $37^{\circ} \mathrm{C}$ in a humidified atmosphere with $5 \% \mathrm{CO}_{2}$. All the cell lines were tested and authenticated.

\section{Generation of ANXA4 knockdown cell lines}

To generate stable ANXA4 knockdown cell lines, RMG-I cells were transfected with a commercial plasmid vector expressing short heparin RNA (shRNA) that targeted ANXA4 mRNA or a negative control nonspecific shRNA (SuperArray Bioscience Corp., KH06928N; Frederick, MD, USA) using Lipofectamine 2000 (Invitrogen, Carlsbad, CA), according to the manufacturer's instructions. After selection using a culture medium containing geneticin ( $600 \mu \mathrm{g} / \mathrm{mL}$; Invitrogen), stable clones were maintained in $250 \mu \mathrm{g} / \mathrm{mL}$ geneticin. Two stable RMG-I-ANXA4 shRNA cell clones were established, designated Y4 and R5 cells. In addition, we transfected the empty vector into the RMG-I cell line using the same procedure to generate control cells, designated NC7. 


\section{Construction of ANXA4 deletion mutants and gene transfection}

Using pcDNA3.1-ANXA4 as a template, fulllength cDNA of ANXA4 was amplified using KODplus (Toyobo Co. Led., Osaka, Japan) with the primers forward 5'-TTGACCTAGAGTCATGGCCA-3', reverse 5'-ATCATCTCCTCCACAGAGAA-3' and subsequently ligated into the pcDNA3.1/V5-His-TOPO vector in-frame with the $\mathrm{C}$-terminal V5 and $6 \times$ His tag. To generate ANXA4 deletion mutants, annexin repeat domains were deleted one by one from the C-terminal site. Three deletion mutants named R3, R2 and R1 were generated and similarly amplified (an Arabic number shows the number of annexin repeat domains). The nucleotide sequences of the forward primers for PCR were the same as those described above for all the deletion mutants, and the reverse primers were as follows: R3 5'-TATAGCCAGCAGAGCATCTT-3', R2 5-CAGAGACACCAGCACTCGCT-3' and R1 5'-CATCCCCACAATCACCTGCT-3'. We subsequently set out to generate an R1 mutant (the E70A mutation), whose calcium-binding site does not work because of the change of a negatively charged carboxyl group to a neutral side chain, as previously described [30]. The sitedirected mutagenesis was performed using the KOD-PlusMutagenesis kit (Toyobo), according to the manufacturer's protocol. These cDNA fragments, including full-length gene, were subsequently inserted between the $B g l$ II and EcoR I sites of the pIRES2AcGFP vector (Clontech, Palo Alto, CA). The sequences of all the mutants were confirmed using the ABI PRISM 3100 Genetic Analyser (Applied Biosystems, Foster City, USA).

Full-length ANXA4, each ANXA4 deletion mutant construct and the empty vector were transfected into NUGC3 cells using Lipofectamine 2000 (Invitrogen). Stable transfectants designated FL-12, R3-6, R2-13, R112, R1(R70A)-95 and NC-14 were obtained by selection in a medium containing geneticin and were maintained in the same manner described above.

\section{Western blotting}

Cells were lysed in RIPA buffer (10 mM Tris- $\mathrm{HCl}$, pH 7.5, $150 \mathrm{mM} \mathrm{NaCl}, 1 \%$ Nonidet P-40, 0.1\% sodium deoxycholate, $0.1 \%$ SDS, $1 \times$ phosphatase inhibitor cocktail (Nacalai Tesque) and $1 \times$ protease inhibitor cocktail (Nacalai Tesque)), followed by centrifugation $\left(13,200 \mathrm{rpm}, 4^{\circ} \mathrm{C}, 15 \mathrm{~min}\right)$. Soluble proteins in the supernatant were separated using sodium dodecyl sulphate polyacrylamide gel electrophoresis, as described previously [28]. Additional information can be found in the Supporting Information on Material and Methods section.

\section{Measurement of $\mathrm{IC}_{50}$ values after treatment with a platinum-based drug}

Cells were suspended in the RPMI medium supplemented with $10 \%$ FBS, seeded in 96-well plates (1,500/well for the RMG-I series and 2,500 cells/well for ANXA4 deletion mutant series), cultured for $24 \mathrm{~h}$ and exposed to various concentrations of cisplatin $(0-25 \mu \mathrm{M}$; Sigma-Aldrich, St Louis, MO) or carboplatin (0-1000 $\mu \mathrm{M}$; Sigma-Aldrich) for $72 \mathrm{~h}$. Cellular proliferation was subsequently evaluated using the WST- 8 assay, i.e. 2-(2-methyosy-4-nitro-phenyl)-3-(4-nitrophenyl)-5-(2,4disulfophynel)-2H-tetrazolium monosodium salt assay (Cell Counting Kit-SF; Nacalai Tesque) after treatment. The absorption of WST-8 was measured at a wavelength of $450 \mathrm{~nm}$ (reference wavelength: $630 \mathrm{~nm}$ ) using a Model 680 microplate reader (Bio-Rad Laboratories, Hercules, CA). Absorbance values for the treated samples were expressed as percentages relative to results for untreated controls, and $\mathrm{IC}_{50}$ values were calculated.

\section{Measurement of intracellular platinum accumulation}

Full-length ANXA4-transfected cells (FL-22), each ANXA4 deletion mutant-transfected cell clone (R3-6, R213, R1-12 and R1[E70A]-95), and control cells (NC-14) were cultured up to $80 \%$ confluence in $150-\mathrm{mm}$ tissue culture dishes. The cells were then exposed to $100 \mu \mathrm{M}$ cisplatin for $60 \mathrm{~min}$ at $37^{\circ} \mathrm{C}$ and washed twice with PBS either immediately or after $3 \mathrm{hr}$ of incubation in cisplatinfree RPMI 1640 medium supplemented with 10\% FBS. Whole-cell extracts were prepared, and the concentration of intracellular platinum was determined using an Agilent 7500ce inductively coupled plasma mass spectrometer (ICP-MS; Agilent, Santa Clara, CA, USA).

\section{In vivo model of cisplatin resistance}

All animal experiments were conducted in accordance with the Institutional Ethical Guidelines for Animal Experimentation of the National Institute of Biomedical Innovation (Osaka, Japan). Female Institute of Cancer Research (ICR) nu/nu mice were obtained from Charles River Japan (Yokohama, Japan). Injection of the ANXA4 knockdown cells was performed as follows: ICR $n u / n u$ mice at 4 weeks of age were subcutaneously inoculated (into the flank of the mice; $\mathrm{n}=6$ per group) with $2.5 \times 10^{6}$ cells of RMG-I NC7 cells or RMG-I-Y4 cells in the total volume of $100 \mu \mathrm{L}$ of 1/1 (v/v) PBS/Matrigel (Becton Dickinson, Bedford, MA). Injection of ANXA4 mutant-transfected cells, i.e. mice at 5 weeks of age were inoculated with $10^{6}$ cells of $\mathrm{NC}-14, \mathrm{FL}-22, \mathrm{R} 1-12$ or R1(E70A) in the same manner 
as with the ANXA4 knockdown cells. Treatment with cisplatin $(3 \mathrm{mg} / \mathrm{kg})$ or PBS i.p. was initiated 1 week after inoculation and administered twice weekly for 4 weeks (ANXA4 knockdown cells) and once a week for 3 weeks (ANXA4 mutant-transfected cells). Tumour volumes were determined twice weekly by measuring length (L), width (W) and depth (D) and using the following formula: tumour volume $\left(\mathrm{mm}^{3}\right)=\mathrm{W} \times \mathrm{L} \times \mathrm{D}$.

\section{$\left[\mathrm{Cl}^{-}\right]_{\mathrm{i}}$ measurements}

$\left[\mathrm{Cl}^{-}\right]_{i}$ was measured using the fluorescent $\mathrm{Cl}^{-}$ indicator N-ethroxycarbonymethyl-6-methoxyquinolinium bromide (MQAE; Dojindo, Kumamoto, Japan). $\left[\mathrm{Cl}^{-}\right]_{\mathrm{i}}$ is detected by the mechanism of diffusion-limited collisional quenching of MQAE fluorescence. MQAE fluorescence intensity inversely correlates with $\left[\mathrm{Cl}^{-}\right]_{\mathrm{i}}$. The cells of the ANXA4 deletion mutant series (NC-14, FL-22, R1-12 and R1[E70A]-95) were cultured in 35-mm tissue culture dishes up to $20 \%$ confluence and incubated with a medium containing $10 \mathrm{mM} \mathrm{MQAE}$ for $4 \mathrm{~h}$ at $37^{\circ} \mathrm{C}$. After loading, the cells were washed 5 times with $\mathrm{Cl}^{-}$-free buffer and electrically stimulated under a microscope at $37^{\circ} \mathrm{C}$ in a humidified atmosphere with $5 \% \mathrm{CO}_{2}$. Fluorescence measurements were initiated immediately at the indicated periods using Biozero BZ-9000 (Keyence, Tokyo, Japan) at $510 / 40 \mathrm{~nm}$ excitation and $380 / 50 \mathrm{~nm}$ emission. The fluorescence was quantitated by means of a standardised procedure using a BZ-II Analyser (Keyence), and the data were presented as the reciprocal of the ratio of fluorescence data (F0/F30) to identify possible correlations with the increase in $\left[\mathrm{Cl}^{-}\right]_{\mathrm{i} .}$.

\section{Statistical analysis}

All calculations involved one-way analysis of variance (ANOVA) followed by Dunnett's analysis to evaluate the significance of differences. In all experiments, $\mathrm{p}$ value of $<0.05$ was considered statistically significant.

\section{ACKNOWLEDGEMENTS}

We thank Y. Kanazawa and A. Yagi for their secretarial assistance, M. Ako and E. Harada for technical assistance.

There are no conflicts of interest to declare. This study was supported by a Grant-in-Aid from the Program for Promotion of Fundamental Studies in Health Sciences of the National Institute of Biomedical Innovation; a Grant-in-Aid from the Ministry of Health, Labour and Welfare of Japan and Grants-in-Aid for Young Scientists (B) (22791560) from the Japanese Ministry of Education, Science, Culture and Sports

\section{REFERENCES}

1. Kelland L. The resurgence of platinum-based cancer chemotherapy. Nat Rev Cancer. 2007; 7(8):573-584.

2. Loriot Y, Mordant P, Deutsch E, Olaussen KA and Soria JC. Are RAS mutations predictive markers of resistance to standard chemotherapy? Nat Rev Clin Oncol. 2009; 6(9):528-534.

3. Alva AS, Matin SF, Lerner SP and Siefker-Radtke AO. Perioperative chemotherapy for upper tract urothelial cancer. Nat Rev Urol. 2012; 9(5):266-273.

4. Bookman MA. First-line chemotherapy in epithelial ovarian cancer. Clin Obstet Gynecol. 2012; 55(1):96-113.

5. Matos CS, de Carvalho AL, Lopes RP and Marques MP. New strategies against prostate cancer--Pt(II)-based chemotherapy. Curr Med Chem. 2012; 19(27):4678-4687.

6. Rossi A, Di Maio M, Chiodini P, Rudd RM, Okamoto H, Skarlos DV, Fruh M, Qian W, Tamura T, Samantas E, Shibata T, Perrone F, Gallo C, Gridelli C, Martelli O and Lee SM. Carboplatin- or cisplatin-based chemotherapy in first-line treatment of small-cell lung cancer: the COCIS meta-analysis of individual patient data. J Clin Oncol. 2012; 30(14):1692-1698.

7. Muggia FM and Los G. Platinum resistance: laboratory findings and clinical implications. Stem Cells. 1993; 11(3):182-193.

8. Jassem J. Chemotherapy of advanced non-small cell lung cancer. Ann Oncol. 1999; 10 Suppl 6:77-82.

9. Perez RP. Cellular and molecular determinants of cisplatin resistance. Eur J Cancer. 1998; 34(10):1535-1542.

10. Fuertes MA, Castilla J, Alonso C and Perez JM. Novel concepts in the development of platinum antitumor drugs. Curr Med Chem Anticancer Agents. 2002; 2(4):539-551.

11. Gonzalez VM, Fuertes MA, Alonso C and Perez JM. Is cisplatin-induced cell death always produced by apoptosis? Mol Pharmacol. 2001; 59(4):657-663.

12. Niedner H, Christen R, Lin X, Kondo A and Howell SB. Identification of genes that mediate sensitivity to cisplatin. Mol Pharmacol. 2001; 60(6):1153-1160.

13. Ishida S, Lee J, Thiele DJ and Herskowitz I. Uptake of the anticancer drug cisplatin mediated by the copper transporter Ctr1 in yeast and mammals. Proc Natl Acad Sci U S A. 2002; 99(22):14298-14302.

14. Safaei R, Holzer AK, Katano K, Samimi G and Howell SB. The role of copper transporters in the development of resistance to Pt drugs. J Inorg Biochem. 2004; 98(10):16071613.

15. Katano K, Kondo A, Safaei R, Holzer A, Samimi G, Mishima M, Kuo YM, Rochdi $M$ and Howell SB. Acquisition of resistance to cisplatin is accompanied by changes in the cellular pharmacology of copper. Cancer Res. 2002; 62(22):6559-6565.

16. Samimi G, Varki NM, Wilczynski S, Safaei R, Alberts DS and Howell SB. Increase in expression of the copper 
transporter ATP7A during platinum drug-based treatment is associated with poor survival in ovarian cancer patients. Clin Cancer Res. 2003; 9(16 Pt 1):5853-5859.

17. Rabik CA, Maryon EB, Kasza K, Shafer JT, Bartnik CM and Dolan ME. Role of copper transporters in resistance to platinating agents. Cancer Chemother Pharmacol. 2009; 64(1):133-142.

18. Koike K, Kawabe T, Tanaka T, Toh S, Uchiumi T, Wada M, Akiyama S, Ono M and Kuwano M. A canalicular multispecific organic anion transporter (cMOAT) antisense cDNA enhances drug sensitivity in human hepatic cancer cells. Cancer Res. 1997; 57(24):5475-5479.

19. Cui Y, Konig J, Buchholz JK, Spring H, Leier I and Keppler D. Drug resistance and ATP-dependent conjugate transport mediated by the apical multidrug resistance protein, MRP2, permanently expressed in human and canine cells. Mol Pharmacol. 1999; 55(5):929-937.

20. Liedert B, Materna V, Schadendorf D, Thomale J and Lage H. Overexpression of cMOAT (MRP2/ABCC2) is associated with decreased formation of platinum-DNA adducts and decreased G2-arrest in melanoma cells resistant to cisplatin. J Invest Dermatol. 2003; 121(1):172-176.

21. Ban N, Takahashi Y, Takayama T, Kura T, Katahira T, Sakamaki S and Niitsu Y. Transfection of glutathione S-transferase (GST)-pi antisense complementary DNA increases the sensitivity of a colon cancer cell line to adriamycin, cisplatin, melphalan, and etoposide. Cancer Res. 1996; 56(15):3577-3582.

22. Olaussen KA, Dunant A, Fouret P, Brambilla E, Andre F, Haddad V, Taranchon E, Filipits M, Pirker R, Popper HH, Stahel R, Sabatier L, Pignon JP, Tursz T, Le Chevalier T and Soria JC. DNA repair by ERCC1 in non-small-cell lung cancer and cisplatin-based adjuvant chemotherapy. N Engl J Med. 2006; 355(10):983-991.

23. Wang Q, Shi S, He W, Padilla MT, Zhang L, Wang X, Zhang B and Lin Y. Retaining MKP1 expression and attenuating JNK-mediated apoptosis by RIP1 for cisplatin resistance through miR-940 inhibition. Oncotarget. 2014; 5(5):1304-1314.

24. Li $\mathrm{H}$ and Yang BB. MicroRNA in drug resistance. Oncoscience. 2014; 1(1):2.

25. Yang H, Kong W, He L, Zhao JJ, O’Donnell JD, Wang J, Wenham RM, Coppola D, Kruk PA, Nicosia SV and Cheng JQ. MicroRNA expression profiling in human ovarian cancer: miR-214 induces cell survival and cisplatin resistance by targeting PTEN. Cancer Res. 2008; 68(2):425433.

26. Sorrentino A, Liu CG, Addario A, Peschle C, Scambia G and Ferlini C. Role of microRNAs in drug-resistant ovarian cancer cells. Gynecol Oncol. 2008; 111(3):478-486.

27. Perego P, Giarola M, Righetti SC, Supino R, Caserini C, Delia D, Pierotti MA, Miyashita T, Reed JC and Zunino F. Association between cisplatin resistance and mutation of p53 gene and reduced bax expression in ovarian carcinoma cell systems. Cancer Res. 1996; 56(3):556-562.

28. Kim A, Enomoto T, Serada S, Ueda Y, Takahashi T, Ripley B, Miyatake T, Fujita M, Lee CM, Morimoto K, Fujimoto M, Kimura T and Naka T. Enhanced expression of Annexin A4 in clear cell carcinoma of the ovary and its association with chemoresistance to carboplatin. Int J Cancer. 2009; 125(10):2316-2322.

29. Gerke V and Moss SE. Annexins: from structure to function. Physiol Rev. 2002; 82(2):331-371.

30. Nelson MR and Creutz CE. Combinatorial mutagenesis of the four domains of annexin IV: effects on chromaffin granule binding and aggregating activities. Biochemistry. 1995; 34(9):3121-3132.

31. Kaetzel MA, Hazarika P and Dedman JR. Differential tissue expression of three $35-\mathrm{kDa}$ annexin calcium-dependent phospholipid-binding proteins. J Biol Chem. 1989; 264(24):14463-14470.

32. Chan HC, Kaetzel MA, Gotter AL, Dedman JR and Nelson DJ. Annexin IV inhibits calmodulin-dependent protein kinase II-activated chloride conductance. A novel mechanism for ion channel regulation. J Biol Chem. 1994; 269(51):32464-32468.

33. Kaetzel MA, Mo YD, Mealy TR, Campos B, BergsmaSchutter W, Brisson A, Dedman JR and Seaton BA. Phosphorylation mutants elucidate the mechanism of annexin IV-mediated membrane aggregation. Biochemistry. 2001; 40(13):4192-4199.

34. Dreier R, Schmid KW, Gerke V and Riehemann K. Differential expression of annexins I, II and IV in human tissues: an immunohistochemical study. Histochem Cell Biol. 1998; 110(2):137-148.

35. Wei R, Zhang Y, Shen L, Jiang W, Li C, Zhong M, Xie Y, Yang D, He L and Zhou Q. Comparative proteomic and radiobiological analyses in human lung adenocarcinoma cells. Mol Cell Biochem. 2012; 359(1-2):151-159.

36. Alfonso P, Canamero M, Fernandez-Carbonie F, Nunez A and Casal JI. Proteome analysis of membrane fractions in colorectal carcinomas by using 2D-DIGE saturation labeling. J Proteome Res. 2008; 7(10):4247-4255.

37. Zimmermann U, Balabanov S, Giebel J, Teller S, Junker H, Schmoll D, Protzel C, Scharf C, Kleist B and Walther $\mathrm{R}$. Increased expression and altered location of annexin IV in renal clear cell carcinoma: a possible role in tumour dissemination. Cancer Lett. 2004; 209(1):111-118.

38. Sitek B, Luttges J, Marcus K, Kloppel G, Schmiegel W, Meyer HE, Hahn SA and Stuhler K. Application of fluorescence difference gel electrophoresis saturation labelling for the analysis of microdissected precursor lesions of pancreatic ductal adenocarcinoma. Proteomics. 2005; 5(10):2665-2679.

39. Xin W, Rhodes DR, Ingold C, Chinnaiyan AM and Rubin MA. Dysregulation of the annexin family protein family is associated with prostate cancer progression. Am J Pathol. 2003; 162(1):255-261. 
40. Duncan R, Carpenter B, Main LC, Telfer C and Murray GI. Characterisation and protein expression profiling of annexins in colorectal cancer. Br J Cancer. 2008; 98(2):426433.

41. Yamashita T, Nagano K, Kanasaki S, Maeda Y, Furuya T, Inoue M, Nabeshi H, Yoshikawa T, Yoshioka Y, Itoh N, Abe Y, Kamada H, Tsutsumi Y and Tsunoda S. Annexin A4 is a possible biomarker for cisplatin susceptibility of malignant mesothelioma cells. Biochem Biophys Res Commun. 2012; 421(1):140-144.

42. Choi $\mathrm{CH}$, Sung CO, Kim HJ, Lee YY, Song SY, Song T, Kim J, Kim TJ, Lee JW, Bae DS and Kim BG. Overexpression of annexin A4 is associated with chemoresistance in papillary serous adenocarcinoma of the ovary. Hum Pathol. 2013; 44(6):1017-1023.

43. Mogami T, Yokota N, Asai-Sato M, Yamada R, Koizume S, Sakuma Y, Yoshihara M, Nakamura Y, Takano Y, Hirahara F, Miyagi $\mathrm{Y}$ and Miyagi E. Annexin a4 is involved in proliferation, chemo-resistance and migration and invasion in ovarian clear cell adenocarcinoma cells. PLoS One. 2013; 8(11):e80359.

44. Matsuzaki S, Enomoto T, Serada S, Yoshino K, Nagamori S, Morimoto A, Yokoyama T, Kim A, Kimura T, Ueda Y, Fujita M, Fujimoto M, Kanai Y and Naka T. Annexin A4conferred platinum resistance is mediated by the copper transporter ATP7A. Int J Cancer. 2013.

45. Jeon YJ, Kim DH, Jung H, Chung SJ, Chi SW, Cho S, Lee SC, Park BC, Park SG and Bae KH. Annexin A4 interacts with the NF-kappaB p50 subunit and modulates NF-kappaB transcriptional activity in a $\mathrm{Ca} 2+$-dependent manner. Cell Mol Life Sci. 2010; 67(13):2271-2281.

46. Al-Bahlani S, Fraser M, Wong AY, Sayan BS, Bergeron R, Melino G and Tsang BK. P73 regulates cisplatin-induced apoptosis in ovarian cancer cells via a calcium/calpaindependent mechanism. Oncogene. 2011; 30(41):4219-4230.

47. Salerno M, Yahia D, Dzamitika S, de Vries E, Pereira-Maia $\mathrm{E}$ and Garnier-Suillerot A. Impact of intracellular chloride concentration on cisplatin accumulation in sensitive and resistant GLC4 cells. J Biol Inorg Chem. 2009; 14(1):123132.

48. Chao AC, Dix JA, Sellers MC and Verkman AS. Fluorescence measurement of chloride transport in monolayer cultured cells. Mechanisms of chloride transport in fibroblasts. Biophys J. 1989; 56(6):1071-1081.

49. Masuishi Y, Arakawa N, Kawasaki H, Miyagi E, Hirahara F and Hirano H. Wild-type p53 enhances annexin IV gene expression in ovarian clear cell adenocarcinoma. FEBS J. 2011; 278(9):1470-1483.

50. Splettstoesser F, Florea AM and Busselberg D. IP(3) receptor antagonist, 2-APB, attenuates cisplatin induced $\mathrm{Ca} 2+$-influx in HeLa-S3 cells and prevents activation of calpain and induction of apoptosis. Br J Pharmacol. 2007; 151(8):1176-1186.

51. Safaei R. Role of copper transporters in the uptake and efflux of platinum containing drugs. Cancer Lett. 2006; 234(1):34-39.

52. El-Khateeb M, Appleton TG, Charles BG and Gahan LR. Development of HPLC conditions for valid determination of hydrolysis products of cisplatin. J Pharm Sci. 1999; 88(3):319-326.

53. Kelland LR. Preclinical perspectives on platinum resistance. Drugs. 2000; 59 Suppl 4:1-8; discussion 37-38.

54. Siddik ZH. Cisplatin: mode of cytotoxic action and molecular basis of resistance. Oncogene. 2003; 22(47):7265-7279.

55. Gaudio E, Paduano F, Spizzo R, Ngankeu A, Zanesi N, Gaspari M, Ortuso F, Lovat F, Rock J, Hill GA, Kaou M, Cuda G, Aqeilan RI, Alcaro S, Croce CM and Trapasso F. Fhit delocalizes annexin a4 from plasma membrane to cytosol and sensitizes lung cancer cells to Paclitaxel. PLoS One. 2013; 8(11):e78610 